\title{
Commentary
}

\section{The Origin of Renal Fibroblasts and Progression of Kidney Disease}

\author{
H. Terence Cook \\ From the Department of Histopathology, Imperial College, \\ London, United Kingdom
}

Regardless of the initial cause of renal injury, failing kidneys approaching end-stage disease typically show marked tubular atrophy and interstitial fibrosis. In addition, in both animal models and humans, once the number of functioning nephrons falls below a critical level, there is an inexorable progression of further nephron loss, tubular atrophy, and interstitial fibrosis. Therefore, it is clearly of importance to understand the pathophysiology of this process. Recent research on the progression of renal disease has centered on three related but independent hypotheses:

1. The final common pathway of renal injury is interstitial fibrosis and therefore preventing interstitial fibrosis will slow the progressive decline in renal function.

2. A major source of the fibroblasts that produce interstitial collagen in the injured kidney is tubular epithelial cells that have differentiated to fibroblasts and crossed the basement membrane. This process has been referred to as epithelial-mesenchymal transition (EMT) and elucidation of this process has been a major focus of research over the past decade. The belief is that understanding this process will allow interventions that will slow interstitial fibrosis.

3. In glomerular disease, proteinuria is a major stimulus to the alteration of epithelial cell function and leads to EMT and interstitial fibrosis.

These three hypotheses have been widely accepted and, indeed, in most review articles the process of EMT is often stated as fact. However, as with all hypotheses, it is important to continue to test them with the best methods available. In this issue of The American Journal of Pathology, Humphreys et al ${ }^{1}$ have performed elegant studies that cast considerable doubt on the existence of EMT in the kidney.

They have used genetic manipulation to develop mice that express protein labels exclusively in renal tubular epithelial cells. They then induced injury in the kidney and, by looking for labeled cells, were able to determine whether any tubular cells had moved into the interstitium or had acquired the ability to behave as fibroblasts.

Using the cre/lox technique to label cells, which depends on crossing mice that express the enzyme cre-recombinase under the control of a cell-lineage specific promoter with mice expressing a reporter gene that is activated by cre-recombinase, Humphreys et al ${ }^{1}$ generated two types of mice: one in which all tubular epithelial cells except those in the collecting duct were labeled and one in which collecting duct cells were labeled. The authors then subjected these mice to two types of injury that led to interstitial fibrosis: unilateral ureteric obstruction (UUO) and ischemia-reperfusion injury. Histological examination of the kidneys postinjury revealed interstitial fibrosis with many cells in the interstitium expressing $\alpha$-smooth muscle actin $(\alpha$-SMA), a marker of myofibroblasts. However, the authors did not find any labeled tubular epithelial cells in the interstitium nor any tubular cells that expressed $\alpha$-smooth muscle actin, implying that EMT did not occur.

Humphreys et al ${ }^{1}$ performed a number of critical controls. One possible explanation for their data is that the marker protein is not expressed by fibroblast-like cells. They therefore used two different marker proteins and showed the same results with both. In addition, they examined mice that expressed the marker protein $\beta$-galactosidase in all cells and showed that it continued to be expressed by the interstitial cells in their model. They also studied whether the labeled epithelial cells could undergo changes in cell culture that other investigators have considered part of the process of EMT. They confirmed this by showing that when the cells were incubated with transforming growth factor- $\beta$, they expressed $\alpha$-SMA and S100A4, markers that have generally been considered as evidence of EMT, while also continuing to express the marker protein.

Thus, this study provides strong evidence against the process of EMT having a significant involvement in interstitial fibrosis in two models of renal fibrosis and comple-

Accepted for publication September 17, 2009

Address reprint requests to $\mathrm{H}$. Terence Cook, FMedSci, Department of Histopathology, Imperial College, Du Cane Rd, London W12 ONN, UK. E-mail: t.cook@imperial.ac.uk. 
ments other studies that have also cast doubt on its existence. In one such study, Faulkner et $\mathrm{al}^{2}$ studied an accelerated model of angiotensin II-induced renal fibrosis and found no evidence for an epithelial origin for interstitial fibroblasts.

So where do the interstitial fibroblasts come from in these models? Another theory that has attracted support is that some interstitial fibroblasts are derived from circulating cells of bone marrow origin. Our group tested this hypothesis by using a mouse that expressed a reporter gene under the control of the promoter of the $\alpha$-2 chain of type I collagen and found no evidence that cells from the bone marrow could synthesize collagen in the kidney after unilateral ureteric obstruction. ${ }^{3}$ Similarly, Lin et al ${ }^{4}$ failed to find a significant contribution of circulating cells to renal fibroblasts by using the promoter of the $\alpha-1$ chain of type I collagen. In fact, it appears that the major source of interstitial collagen-producing cells is from fibroblasts that reside within the interstitium itself and particularly from cells in the adventitia of arterioles and arteries. ${ }^{2,5}$ In agreement with this, Humphreys et $\mathrm{al}^{1}$ also now show that cells of metanephric mesenchymal origin expressing platelet-derived growth factor receptor $\beta$ (PDGFR $\beta$ ), consistent with pericytes, are the major source of interstitial fibroblasts in unilateral ureteric obstruction.

If it is true that EMT does not contribute significantly to interstitial fibrosis, and I think the evidence against EMT is now strong, one has to ask why this theory had achieved such widespread support and what is the evidence in its favor. In part it is because the expression epithelial mesenchymal transition has been applied to a range of phenomena, and there has been confusion as to how the term is used. At one end of the spectrum it has been used to describe changes that renal tubular epithelial cells can show in culture whereby they express makers such as $\alpha$-SMA that are more typical of cells of mesenchymal origin. That this occurs is uncontroversial and indeed Humphreys et $\mathrm{al}^{1}$ show that their labeled epithelial cells express $\alpha$-SMA after stimulation with transforming growth factor- $\beta$. It is also clear that when renal tubular cells are damaged in vivo they undergo morphological changes of dedifferentiation and may sometimes express markers such as $\alpha$-SMA. ${ }^{6}$ However, the demonstration of these changes falls a long way short of what is usually meant by EMT, which is that these cells traverse the basement membrane and start to synthesize type I collagen. I think the evidence in favor of this is much more tenuous.

A great deal of weight has been placed on the expression by tubular epithelial cells of a protein that has been referred to as fibroblast specific protein 1, which is a form of S100 protein, S100A4. ${ }^{7}$ Indeed, one of the major articles supporting a role for both tubular epithelial cells and circulating cells differentiating to interstitial fibroblasts relies mainly on the co-expression of S100A4. ${ }^{8}$ In that article, the authors used a lineage-specific marker for tubular epithelial cells and showed that in UUO there was cellular co-localization of the tubular marker and S100A4. However, the expression of this protein by epithelial cells inside the tubular basement membrane is no more evidence for their ability to become interstitial fibroblasts than is expression of another marker such as $\alpha$-SMA. In addition, there is evidence that S100A4 can be expressed by leukocytes in injured kidneys, ${ }^{7}$ by podocytes in diabetic nephropathy, ${ }^{9}$ and by many other cell types including human monocytes and macrophages (reviewed in Mazzucchelli ${ }^{10}$ ), and so it is very doubtful whether it can be considered a fibroblast-specific protein.

In summary, it is clear that when renal tubular epithelial cells are injured they undergo morphological and biochemical changes and may express proteins that can also be expressed by cells of mesenchymal origin. This is likely to be related to their ability to repair and regenerate after acute tubular damage. However, as the study by Humphreys et $\mathrm{al}^{1}$ demonstrates, there is little evidence that these altered cells can move into the interstitium and produce collagen, and the main collagen-producing cells are intrinsic renal cells, particularly perivascular cells. However, this does not exclude a possible role for communication between the damaged epithelial cells and interstitial cells that may be important in stimulating fibrosis.

If EMT does not occur, what about the other two hypotheses mentioned earlier? I think these also need critical examination. The first states that the final common pathway of renal injury is interstitial fibrosis and therefore preventing interstitial fibrosis will slow the progressive decline in renal function. This is often stated as if it is self-evident. However, while it is obvious that almost all severely damaged kidneys show marked fibrosis, it is much less clear that preventing the fibrosis would have prevented loss of renal function. Morphologically, fibrosis is seen in association with atrophic tubules. It is likely that the fibrosis is a secondary response to the tubular damage and that preventing fibrosis would not necessarily alter the tubular degeneration. This is particularly relevant to glomerular diseases where glomerular scarring, if it involves the origin of the proximal tubule, leads to secondary tubular atrophy and fibrosis around the atrophic tubule. However, fibrosis in that case is a secondary response and preventing it would not alter the tubular damage.

As has been pointed out by Kriz and LeHir, ${ }^{11}$ in a very thorough review, the hypothesis that fibrosis itself leads to progressive decline in renal function is dependent on fibrosis around a damaged nephron then causing injury to adjacent nephrons, but there is no experimental evidence for this. Indeed, careful morphological studies ${ }^{12}$ show that it is only in nephrons with irreversible glomerular injury that the tubules appear damaged with no evidence of spread to adjacent tubules. Thus, in glomerular disease at least, there is no evidence for progression of the disease at the level of the tubulointerstitium. Indeed, it may be that by replacing damaged nephrons with scar tissue the interstitial reaction confines the degenerative change to the damaged nephron. This does not exclude a role for interstitial fibrosis in progression of other processes such as transplant rejection but does emphasize the need for more careful evaluation of pathophysiology in different settings.

What about the third hypothesis that in glomerular disease, proteinuria is a major stimulus to alteration of epithelial cell function and leads to EMT and interstitial fibrosis? Even if we accept that EMT does not occur, it is plausible that tubular cells damaged by protein, or other 
substances in the tubular lumen, might signal to cells in the interstitium and that this might stimulate interstitial fibroblasts to lay down collagen. ${ }^{13}$ This would also fit with the fact that the magnitude of proteinuria correlates well with the rate of progression of renal disease. However, much of the experimental evidence that has been put forward to support this is consistent with other mechanisms, and there are several authors who argue against its importance as discussed in detail in the review by Kriz and Le Hir. ${ }^{11}$ Careful analysis of experimental glomerular injury suggests that, even with severe glomerular injury, the proximal tubule remains healthy unless the glomerular damage encroaches on the glomerulotubular junction. In agreement with this, abrogation of protein uptake into proximal tubules in transgenic mice with a kidney-specific deficiency of megalin did not alter tubular degenerative changes in crescentic glomerulonephritis and, in fact, megalin-deficient tubular cells showed increased apoptosis. $^{14}$

In conclusion, I believe that all three of the hypotheses with which I began this commentary need careful re-evaluation. Their uncritical acceptance risks diverting research efforts from other areas that are potentially of more importance in the progression of renal disease including the control of scarring versus repair in glomeruli, and the role of the peritubular capillaries. The article by Humphreys et $\mathrm{al}^{1}$ is an important step in this re-evaluation.

\section{References}

1. Humphreys BD, Lin S-J, Kobayashi A, Hudson TE, Nowlin BT, Bonventre JV, Valerius MT, McMahon AP, Duffield JS: Fate tracing reveals the pericyte and not epithelial origin of myofibroblasts in kidney fibrosis. Am J Pathol 2010, 1:85-97
2. Faulkner JL, Szcykalski LM, Springer F, Barnes JL: Origin of interstitial fibroblasts in an accelerated model of angiotensin II-induced renal fibrosis. Am J Pathol 2005, 167:1193-1205

3. Roufosse C, Bou-Gharios G, Prodromidi E, Alexakis C, Jeffery R, Khan S, Otto WR, Alter J, Poulsom R, Cook HT: Bone marrow-derived cells do not contribute significantly to collagen I synthesis in a murine model of renal fibrosis. J Am Soc Nephrol 2006, 17:775-782

4. Lin SL, Kisseleva T, Brenner DA, Duffield JS: Pericytes and perivascular fibroblasts are the primary source of collagen-producing cells in obstructive fibrosis of the kidney. Am J Pathol 2008, 173:1617-1627

5. Wiggins R, Goyal M, Merritt S, Killen PD: Vascular adventitial cell expression of collagen I messenger ribonucleic acid in anti-glomerular basement membrane antibody-induced crescentic nephritis in the rabbit: a cellular source for interstitial collagen synthesis in inflammatory renal disease. Lab Invest 1993, 68:557-565

6. Ng YY, Huang TP, Yang WC, Chen ZP, Yang AH, Mu W, NikolicPaterson DJ, Atkins RC, Lan HY: Tubular epithelial-myofibroblast transdifferentiation in progressive tubulointerstitial fibrosis in 5/6 nephrectomized rats. Kidney Int 1998, 54:864-876

7. Le HM, Hegyi I, Cueni-Loffing D, Loffing J, Kaissling B: Characterization of renal interstitial fibroblast-specific protein 1/S100A4-positive cells in healthy and inflamed rodent kidneys. Histochem Cell Biol 2005, 123:335-346

8. Iwano M, Plieth D, Danoff TM, Xue C, Okada H, Neilson EG: Evidence that fibroblasts derive from epithelium during tissue fibrosis. J Clin Invest 2002, 110:341-350

9. Yamaguchi Y, Iwano M, Suzuki D, Nakatani K, Kimura K, Harada K, Kubo A, Akai Y, Toyoda M, Kanauchi M, Neilson EG, Saito Y: Epithelialmesenchymal transition as a potential explanation for podocyte depletion in diabetic nephropathy. Am J Kidney Dis 2009, 54:653-664

10. Mazzucchelli L: Protein S100A4: too long overlooked by pathologists? Am J Pathol 2002, 160:7-13

11. Kriz W, Le Hir M: Pathways to nephron loss starting from glomerular diseases-insights from animal models. Kidney Int 2005, 67:404-419

12. Le Hir M, Besse-Eschmann V: A novel mechanism of nephron loss in a murine model of crescentic glomerulonephritis. Kidney Int 2003, 63:591-599

13. Abbate M, Zoja C, Remuzzi G: How does proteinuria cause progressive renal damage? J Am Soc Nephrol 2006, 17:2974-2984

14. LeHir M, Kriz W: New insights into structural patterns encountered in glomerulosclerosis. Curr Opin Nephrol Hypertens 2007, 16:184-191 